\title{
Does biology determine survival in pancreatic cancer?
}

\author{
Savio George Barreto*,1,2 \\ ${ }^{1}$ Division of Surgery \& Perioperative Medicine Flinders Medical Centre, Bedford Park, Adelaide, South Australia, Australia \\ ${ }^{2}$ College of Medicine \& Public Health, Flinders University, Adelaide, South Australia, Australia \\ *Author for correspondence: Tel.: +61 88204 5511; savio.barreto@sa.gov.au
}

First draft submitted: 21 November 2019; Accepted for publication: 10 December 2019; Published online: 24 December 2019

Keywords: morbidity • mortality • outcomes $\bullet$ survival

A recent publication in JAMA Surgery by Jones et al. [1] based on a secondary analysis of the ESPAC-4 data presented an interesting observation. While local recurrences following curative surgery for pancreatic cancer occur significantly later than distant metastases, the authors were unable to find a difference in overall survival between these two patient cohorts. This led the authors to conclude that pancreatic cancer is a systemic disease and all patients must receive adjuvant chemotherapy. The inferences seem fair and corroborate previous evidence from the clinic [2], from computer modeling [3] and experimental mouse models [4].

\section{Pancreatic cancer - a systemic disease at the outset?}

In the last 1 year, among the various publications that have addressed the issue of recurrence of pancreatic cancer following surgery, a meta-analysis exploring patterns of recurrence published by Tanaka et al. [5] is thought provoking. The authors noted that the liver $(26.5 \%)$ was the most common site of metastases after pancreatic cancer surgery followed closely by locoregional recurrences (20.8\%). Additionally, they found that peritoneal dissemination was the site of recurrence with the lowest overall survival (median 14.1 months) with an R1 resection being one of its most significant contributing factors. Intuitively, one would assume that an R1 resection would lead to locoregional recurrence. However, the evidence gets even more confusing. While R1 resections may be associated with a significantly reduced time to loco-regional recurrence in comparison to an R0 resection (median 16 vs 36 months; $\mathrm{p}=0.002$ ) there is no difference in the patterns of recurrence! [6] This suggests that the only major difference between an R0 and R1 resections is the time to development of recurrence or in other words, an R0 resection may only be delaying the inevitable. In fact, our own meta-analysis addressing the role of immediate revision of the surgical margin in patients undergoing R1 resections based on intra-operative frozen section failed to demonstrate a survival advantage in those patients in whom the revision resulted in a margin-negative (R0) resection [7]. These data taken together suggest that pancreatic cancer is likely a systemic disease at presentation and it is the biology of the disease that drives the pattern of recurrence. A good surgery with a margin-negative resection [8], at best, helps slow disease progression. Another study by Tummers et al. [6] appreciated a lack of benefit of an R0 resection on survival in patients with node-positive disease. All these observations lead us to pose the next logical question - 'Can neoadjuvant chemotherapy be used to improve survival in pancreatic cancer?'.

\section{But is not this what happened with breast cancer?}

This takes us back nearly four decades when in 1980 Bernard Fisher boldly challenged contemporary thinking by stating that breast cancer was a systemic disease. This paved the way for neoadjuvant chemotherapy followed by less radical surgery. However, we must be mindful of the evidence that emerged thereafter. Neoadjuvant chemotherapy has no survival advantage over adjuvant chemotherapy. In fact, the only perceived benefit of neoadjuvant chemotherapy, that of downsizing the tumors rendering the patient amenable to breast-conserving surgery, is not without its attendant risks. Patients receiving neoadjuvant chemotherapy and then undergoing breast-conserving surgery have a significantly higher rate (by $5.5 \%$; $<0.0001$ ) of local recurrence at 15 years compared with patients receiving adjuvant therapy [9]. In the pancreas, less radical surgery is not an option and 
neoadjuvant therapy is being trialed in borderline-resectable and locally advanced cancers $[10,11]$. Neoadjuvant chemotherapy is also being evaluated in clearly resectable pancreatic cancer given the negative impact of tumor size $>2 \mathrm{~cm}$ on overall survival [12]. All these indications for the use of neoaodjuvant chemotherapy in pancreatic cancer need to be addressed within the confines of a well-conducted randomized controlled trial (comparing neoadjuvant vs adjuvant chemotherapy).

\section{Biology of pancreatic cancer}

All the above clinical observations strengthen the argument that it is the underlying biology of the cancer that drives outcomes. Over the last few decades there has been a concerted effort to decipher the pathways involved in pancreatic carcinogenesis. We are aware that pancreatic cancer develops through a well-defined pathway arising from the accumulation of molecular alterations, characterized morphologically by precursor lesions with increasing grades of dysplasia called PanIns that eventually progress to the development of invasive adenocarcinoma [13]. Subsequent work [14] enabled the elucidation of the four main driver genes in pancreatic carcinogenesis, namely KRAS, CDKN2A inactivation, TP53 and SMAD4 inactivation. In fact, SMAD4 inactivation has been consistently associated with higher rates of local as well as distant failure following surgery [15] and even in patients receiving adjuvant chemoradiation [16]. Given its association with an increased risk for early tumor dissemination makes it a valid marker to aid decision making (against surgery) in patients who would otherwise require morbid operations based on their tumor burden. Further efforts to clarify the driver genes in pancreatic carcinogenesis using a global genomic analysis [17] not only confirmed that the above four genes were mutated at the highest frequency, but additionally identified 12 core signaling pathways including KRAS, TGF- $\beta$, Wnt/Notch, hedgehog, integrin, JNK and small GTPase signaling pathways in addition to the pathways involved apoptosis, DNA damage control, invasion, homophilic cell adhesion and control of G1/S phase transition.

The Australian Pancreatic Cancer Genome Initiative continues to steadily piece together the complex genomic landscape puzzle of pancreatic carcinogenesis. Using an integrated genomic expression analysis of 456 pancreatic ductal adenocarcinomas they were able to demonstrate four distinct subtypes of pancreatic ductal adenocarcinoma, namely, squamous, pancreatic progenitor, aberrantly differentiated endocrine exocrine and immunogenic types [18].

A bigger thrust, in recent times, has focused on the identification of biomarkers and genetic signatures linked to cancer prognosis and response to therapy. Puleo [19] stratified pancreatic ductal adenocarcinoma based on tumor and microenvironment features using RNA expression array, immunohistochemistry and targeted-capture DNA sequencing with a view to subclassifying tumors to enable type-specific therapy. They subclassified pancreatic cancer into a) high cellularity: pure classical, immune classical and pure basal-like; and b) all cellularities: pure classical, immune classical, pure basal-like, stroma activated and desmoplastic. The pure basal-like subtype was noted to have the worst prognosis.

Spurred on by the promise of success with immunotherapy in pancreatic cancer, Balachandran et al. [20] used whole exome sequencing, immunohistochemical and transcriptional immunoprofiling, computational biophysics and functional assays to identify T-cell antigens in blood and tissue samples obtained from long-term survivors. Based on the genomic profiling and in silico neoantigen prediction, they noted that tumors with both the highest neoantigen number and the most abundant $\mathrm{CD} 8^{+}$T-cell infiltrates, but neither alone, stratified patients with the longest survival thereby serving as a biomarker to guide immunotherapy.

\section{Does cancer location within the pancreas impact outcomes?}

There is also emerging evidence that cancers in the head of the pancreas behave differently (in terms of survival) from those in the body and tail possibly due to differences in biology [21-27]. Some studies report a poorer survival with tumors in the body and tail as compared with those in the head $[21,23]$ while others report quite the contrary [22,28,29]. Dreyer et al. [27] determined that pancreatic head tumor pathways involved more B-cell and CD8-positive T-cell signaling, as opposed to, epithelial-to-mesenchymal transition, inflammation, hypoxia response, metabolic reprogramming, TP53 expression and squamous differentiation noted in tumors of the body and tail. These findings were corroborated, at least in part, by Birnbaum et al. [21] who noted that the genes upregulated in the pancreatic head cancers were those involved in lymphocyte activation and pancreas exocrine functions while the body and tail cancers were accompanied by genes related to keratinocyte differentiation, in line with the enrichment for squamous phenotype. 
There is a need to clarify the relevance and overall contribution of mismatch repair genes and microsatellite instability to the pancreatic carcinogenesis and outcomes of the cancer with a view to determining if this information may help guide patient treatment [30].

\section{On the balance...}

It is in deciphering this complex biological landscape [31], tempered by an appreciation of underlying tumor diversity [32], that will enable us to understand what makes pancreatic cancer one of the deadliest cancers [33] known to man. These efforts may also enable us to develop more effective and, hopefully, less toxic treatments with the overarching aim of improving meaningful survival. Such attempts at subclassification with a view to guiding personalized therapy are certainly well intentioned. However, we are yet to fully elucidate pancreatic carcinogenesis, how the tumor evolves and metastasizes, and what are the drivers of these behavioral patterns. It is only through understanding the disease in its entirety that we can start to make a serious attempt to gain mastery over it.

\section{Financial \& competing interests disclosure}

The authors have no relevant affiliations or financial involvement with any organization or entity with a financial interest in or financial conflict with the subject matter or materials discussed in the manuscript. This includes employment, consultancies, honoraria, stock ownership or options, expert testimony, grants or patents received or pending, or royalties.

No writing assistance was utilized in the production of this manuscript.

\section{References}

1. Jones RP, Psarelli EE, Jackson R et al. Patterns of recurrence after resection of pancreatic ductal adenocarcinoma: a secondary analysis of the ESPAC-4 randomized adjuvant chemotherapy trial. JAMA Surg. doi:10.1001/jamasurg.2019.3337 (2019).

2. Yachida S, Jones S, Bozic I et al. Distant metastasis occurs late during the genetic evolution of pancreatic cancer. Nature 467(7319), 1114-1117 (2010).

3. Haeno H, Gonen M, Davis MB, Herman JM, Iacobuzio-Donahue CA, Michor F. Computational modeling of pancreatic cancer reveals kinetics of metastasis suggesting optimum treatment strategies. Cell 148(1-2), 362-375 (2012).

4. Rhim AD, Mirek ET, Aiello NM et al. EMT and dissemination precede pancreatic tumor formation. Cell 148(1-2), 349-361 (2012).

5. Tanaka M, Mihaljevic AL, Probst P et al. Meta-analysis of recurrence pattern after resection for pancreatic cancer. Br. J. Surg. 106(12), 1590-1601 (2019).

6. Tummers WS, Groen JV, Sibinga Mulder BG et al. Impact of resection margin status on recurrence and survival in pancreatic cancer surgery. Br. J. Surg. 106(8), 1055-1065 (2019).

7. Barreto S, Pandanaboyana S, Ironside N, Windsor J. Does revision of resection margins based on frozen section improve overall survival following pancreatoduodenctomy for pancreatic ductal adenocarcinoma? A meta-analysis. HPB 19, 573-579 (2017).

8. Howard TJ, Krug JE, Yu J et al. A margin-negative R0 resection accomplished with minimal postoperative complications is the surgeon's contribution to long-term survival in pancreatic cancer. J. Gastrointest. Surg. 10(10), 1338-1345; discussion 1345-1336 (2006).

9. Early Breast Cancer Trialists' Collaborative Group (EBCTCG). Long-term outcomes for neoadjuvant versus adjuvant chemotherapy in early breast cancer: meta-analysis of individual patient data from ten randomised trials. Lancet Oncol. 19(1), 27-39 (2018).

10. Klaiber U, Leonhardt CS, Strobel O, Tjaden C, Hackert T, Neoptolemos JP. Neoadjuvant and adjuvant chemotherapy in pancreatic cancer. Langenbecks Arch. Surg. 403(8), 917-932 (2018).

11. Barreto S, Windsor J. Justifying vein resection with pancreatoduodenectomy. Lancet Oncol. 17(3), e118-e124 (2016).

12. Marchegiani G, Andrianello S, Malleo G et al. Does size matter in pancreatic cancer? Reappraisal of tumour dimension as a predictor of outcome beyond the TNM. Ann. Surg. 266(1), 142-148 (2017).

13. Maitra A, Adsay NV, Argani P et al. Multicomponent analysis of the pancreatic adenocarcinoma progression model using a pancreatic intraepithelial neoplasia tissue microarray. Mod. Pathol. 16(9), 902-912 (2003).

14. Yachida S, White C, Naito Y, Zhong Y, Brosnan J. Clinical significance of the genetic landscape of pancreatic cancer and implications for identification of potential long-term survivors. Clin. Cancer Res. 18(22), 6339-6347 (2012).

15. Hruban RH, Adsay NV. Molecular classification of neoplasms of the pancreas. Hum. Pathol. 40(5), 612-623 (2009).

16. Herman JM, Jabbour SK, Lin SH et al. Smad4 loss correlates with higher rates of local and distant failure in pancreatic adenocarcinoma patients receiving adjuvant chemoradiation. Pancreas 47(2), 208-212 (2018).

17. Jones S, Zhang X, Parsons DW et al. Core signaling pathways in human pancreatic cancers revealed by global genomic analyses. Science 321(5897), 1801-1806 (2008).

18. Bailey P, Chang DK, Nones K et al. Genomic analyses identify molecular subtypes of pancreatic cancer. Nature 531(7592), 47-52 (2016). 
19. Puleo F. Stratification of pancreatic ductal adenocarcinomas based on tumor and microenvironment features. Gastroenterology 155 , 1999-2013 (2018).

20. Balachandran VP, Luksza M, Zhao JN et al. Identification of unique neoantigen qualities in long-term survivors of pancreatic cancer. Nature 551(7681), 512-516 (2017).

21. Birnbaum D, Bertucci F, Finetti P, Birnbaum D, Mamessier E. Head and body/tail pancreatic carcinomas are not the same tumors. Cancers 11(4), 497 (2019).

22. Ling Q, Xu X, Zheng SS, Kalthoff $H$. The diversity between pancreatic head and body/tail cancers: clinical parameters and in vitro models. Hepatobiliary Pancreat. Dis. Int. 12(5), 480-487 (2013).

23. Tomasello G, Ghidini M, Costanzo A et al. Outcome of head compared to body and tail pancreatic cancer: a systematic review and meta-analysis of 93 studies. J. Gastrointest. Oncol. 10(2), 259-269 (2019).

24. Lau MK, Davila JA, Shaib YH. Incidence and survival of pancreatic head and body and tail cancers: a population-based study in the United States. Pancreas 39(4), 458-462 (2010).

25. Watanabe I, Sasaki S, Konishi M et al. Onset symptoms and tumor locations as prognostic factors of pancreatic cancer. Pancreas 28(2), 160-165 (2004).

26. Artinyan A, Soriano PA, Prendergast C, Low T, Ellenhorn JD, Kim J. The anatomic location of pancreatic cancer is a prognostic factor for survival. HPB 10(5), 371-376 (2008).

27. Dreyer SB, Jamieson NB, Upstill-Goddard R et al. Defining the molecular pathology of pancreatic body and tail adenocarcinoma. $B r . J$. Surg. 105(2), e183-e191 (2018).

28. van Erning FN, MacKay TM, van der Geest LGM et al. Association of the location of pancreatic ductal adenocarcinoma (head, body, tail) with tumor stage, treatment, and survival: a population-based analysis. Acta Oncol. 57(12), 1655-1662 (2018).

29. Winer LK, Dhar VK, Wima K et al. The impact of tumor location on resection and survival for pancreatic ductal adenocarcinoma. J. Surg. Res. 239, 60-66 (2019).

30. Hu ZI, Shia J, Stadler ZK et al. Evaluating mismatch repair deficiency in pancreatic adenocarcinoma: challenges and recommendations. Clin. Cancer Res. 24(6), 1326-1336 (2018).

31. Makohon-Moore A, Iacobuzio-Donahue CA. Pancreatic cancer biology and genetics from an evolutionary perspective. Nat. Rev. Cancer 16(9), 553-565 (2016).

32. Perry EB, Makohon-Moore A, Zheng C et al. Tumor diversity and evolution revealed through RADseq. Oncotarget 8(26), 41792-41805 (2017).

33. Maisonneuve P. Epidemiology and burden of pancreatic cancer. Presse Med. 48(3 Pt 2), e113-e123 (2019). 